\title{
Polyamine-Polymeric Micelle Hybrid Hydrogel: Microscopic Properties of Crosslinkers Affecting Macroscopic Rheological Properties of Hydrogel
}

\author{
Chikara Yoshida, Tomoki Ito, Ryosuke Anzai, Kengo Fukuda, Keita Kinoshita, \\ Seiichi Sonotaki, Taku Takami, Yoshihiko Murakami \\ Department of Organic and Polymer Materials Chemistry, Faculty of Engineering, Tokyo University of \\ Agriculture and Technology, Tokyo, Japan \\ Email: muray@cc.tuat.ac.jp
}

Received 24 November 2014; revised 31 December 2014; accepted 5 January 2015

Copyright (C) 2015 by authors and Scientific Research Publishing Inc.

This work is licensed under the Creative Commons Attribution International License (CC BY). http://creativecommons.org/licenses/by/4.0/

(c) (i) Open Access

\begin{abstract}
We have developed a hybrid hydrogel that is formed from a crosslinkable polymeric micelle and a polyamine. Under optimal conditions, the hydrogel rapidly formed in one second after a crosslinkable polymeric micelle solution was mixed with a polyamine solution. We could change the hydrogel's gelation properties, such as the storage modulus and gelation time by tuning the molecular weights of block copolymers and by tuning the $\mathrm{pH}$ of the dissolving-solvent of the hydrogel's constituent components. Furthermore, we have clarified here that the structural difference among the micelles acting as crosslinkers can affect the gelation properties of the hydrogel. According to our findings, the hydrogel that was formed from the polymeric micelles possessing a highly packed (i.e., well-entangled or crosslinked) inner core exhibited a higher storage modulus than the hydrogel that was formed from the polymeric micelles possessing a lowly packed structure. Our results demonstrate that a microscopic structural difference among crosslinkers can induce a macroscopic change in the properties of the resulting hydrogels. For medical applications, the hydrogel proposed in the present paper can encapsulate the hydrophobic compounds in crosslinkers (polymeric micelles) so that the hydrogel can be available as the biomaterial for their sustained release.
\end{abstract}

\section{Keywords}

Hydrogel, Polymeric Micelle, Self-Assembly, Crosslinker, Block Copolymer 


\section{Introduction}

Currently, several tissue-adhesive biomaterials have been proposed for surgical applications. These materials have included fibrin glues [1] [2], collagen sheets with fibrin glues [3], fibrillar collagen [4], collagen with citric acid derivative [5], gelatin with resorcin and formalin [6], albumin with glutaraldehyde [7], cyanoacrylate [8], and synthetic polymers [9]-[12]. However, these materials have a number of disadvantages and need to be improved for clinical use. The potential risk of infectious contamination is a major drawback for fibrin-, collagenand gelatin-based glues [13]-[16]. The glues that contain low-molecular-weight aldehydes (e.g. formaldehyde and glutaraldehyde) as crosslinkers are extremely cytotoxic because they can penetrate into tissues owing to their highly diffusive nature [17] [18]. Synthetic polymer-based glues have been actively developed because they do not pose any risk of infectious contaminations. Essential properties for the synthetic glues are absorbability, biodegradability, non-toxicity, and reactivity in physiological conditions. The molecular design of polymers can easily control these properties. However, complicated preparation procedures [9]-[12] required for some of the synthetic glues make their application difficult.

To resolve these problems, we developed a novel hydrogel that covalently contains both a reactive polymeric micelle formed from poly (ethylene-glycol)- $b$-poly (lactic acid) (PEG-PLA) block copolymers serving as a crosslinker and a polyamine as a main chain of the hydrogel [19]-[24] (Figure 1). PEG-PLA has been widely used for the construction of biomaterials owing to its biodegradability, biocompatibility and proper hydrophilicity. A highly hydrophilic polymer, poly(ethylene glycol) (PEG) has served as a non-inflammatory and non-immunogenic modifier for proteins and drugs. The polymers and materials modified with PEG were generally found to be less thrombogenic than unmodified polymers and materials, owing to the flexible backbone and hydrophilicity of PEG [25]-[31]. Our hydrogel rapidly formed when a polymeric micelle and a polyamine were mixed, and the hydrogel adhered to tissue according to the Schiff base formation between the aldehyde group on the surface of the polymeric micelle and the amino group present on the tissue surface. All polymers forming the hydrogel were synthetic, so there was no risk of critical illness, such as a viral infection or anaphylactic shock. Furthermore, the polymeric micelle could encapsulate hydrophobic compounds into the hydrophobic PLA core of the micelle, thereby allowing the hydrogel to release the hydrophobic compounds in a sustained manner. However, in constructing advanced tissue-adhesive biomaterials, we have yet to address the effects that both macromolecular crosslinkers and the main chain can have on the resulting hydrogel's properties.

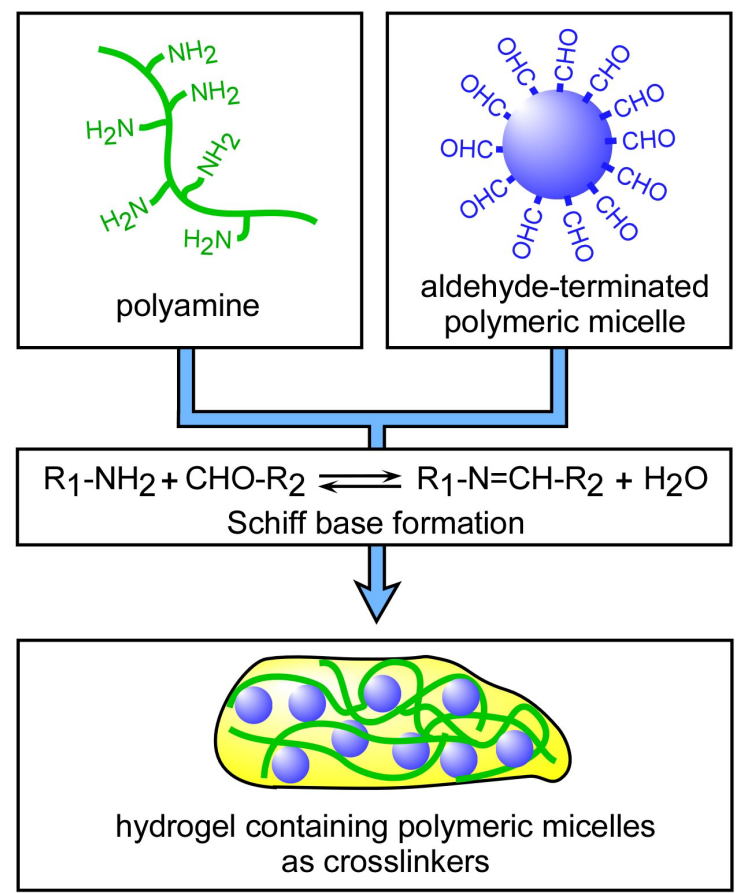

Figure 1. The formation of the hydrogel containing polymeric micelles as crosslinkers. 
For the present paper, we evaluated the effects that both macromolecular crosslinkers and main chain can have on the rheological properties of the resulting hydrogel. One can change the hydrogel's gelation properties, such as the storage modulus and the gelation time by tuning the molecular weights of block copolymers and by tuning the $\mathrm{pH}$ of the dissolving-solvent of the hydrogel's constituent components. Furthermore, we have clarified that the structural difference among the micelles acting as crosslinkers can affect the hydrogel's gelation properties. According to our findings, the hydrogel that was formed from the polymeric micelles possessing a highly packed (i.e., well-entangled or crosslinked) inner core exhibited a higher storage modulus than the hydrogel that was formed from the polymeric micelles possessing a lowly packed structure. Our results demonstrate that a microscopic structural difference among crosslinkers can induce a macroscopic change in the properties of the resulting hydrogels.

\section{Materials and Methods}

\subsection{Materials}

Ethylene oxide (Sumitomo Seika Chemicals Co., Osaka, Japan) was purified by distillation in the presence of CaH2. DL-Lactide (Tokyo Chemical Industry Co., Tokyo, Japan) was recrystallized twice from ethyl acetate. 3,3-Diethoxypropanol was distilled with sodium under reduced pressure. Potassium naphthalene was synthesized by a mixing of potassium and naphthalene in anhydrous tetrahydrofuran (THF) for $18 \mathrm{~h}$. Polyethyleneimine (PEI) with average molecular weights of 1800, 10,000, and 70,000 was generously provided by Nippon Shokubai Co., (Osaka, Japan). All other reagents were of analytical grade and were used without further purification.

\subsection{Synthesis of PEG-PLA Block Copolymer Having Acetal- and Hydroxyl Termini (Acetal-PEG- $b$-PLA-OH)}

PEG-PLA block copolymers having acetal- and hydroxyl termini (acetal-PEG-b-PLA-OH) were synthesized by means of the ring-opening polymerization of both ethylene oxide and ${ }_{\mathrm{DL}}$-lactide in anhydrous THF (Figure 2). For $1 \mathrm{~h}, 3,3$-diethoxypropanol and potassium naphthalene were mixed in THF. The purified ethylene oxide was then added to the obtained potassium 3,3-diethoxypropioxide solution $(50 \mathrm{ml})$, and polymerization was carried

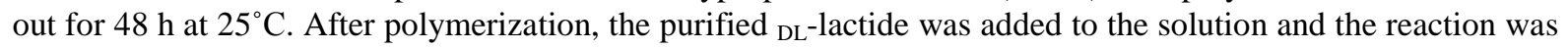
performed for $24 \mathrm{~h}$. After polymerization of $\mathrm{DL}^{-}$-lactide, acetic acid was added to the solution, and the reaction

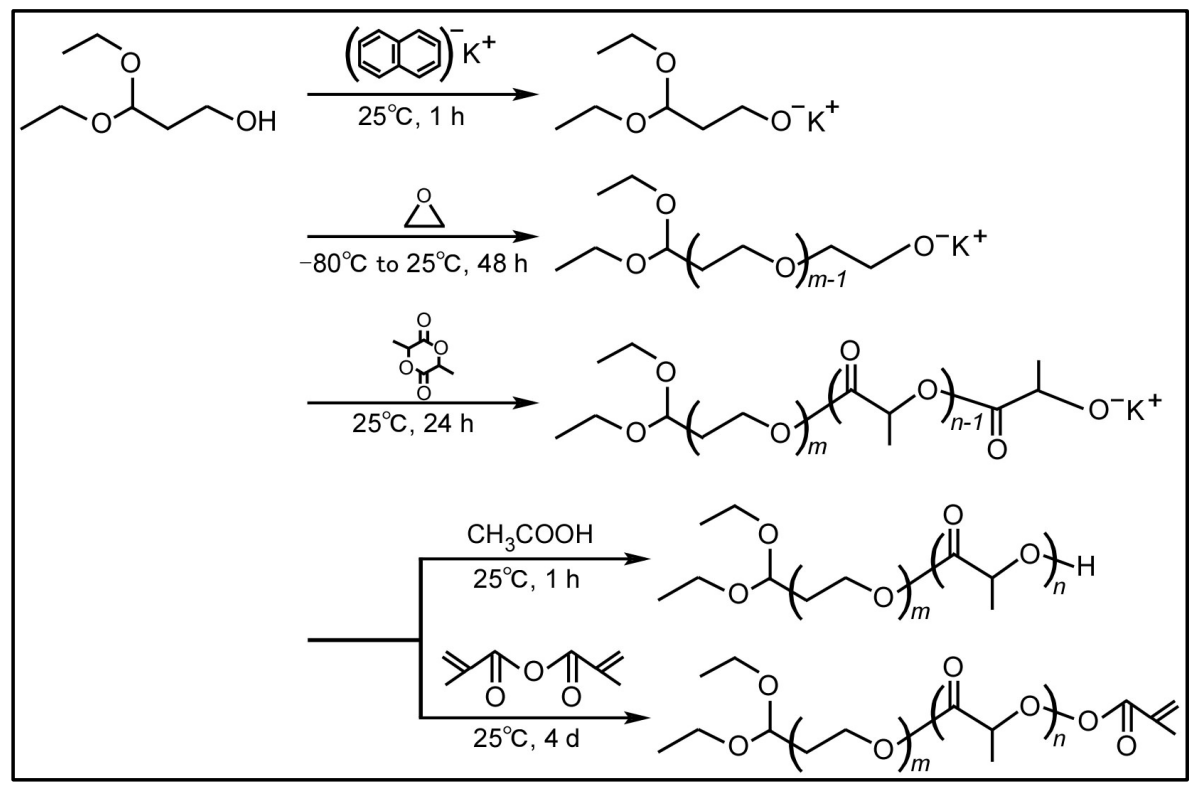

Figure 2. The scheme for the synthesis of PEG-PLA block copolymer having acetal-and hydroxyl termini (acetal-PEG-b-PLA-OH) and PEG-PLA block copolymer having acetal-and vinyl termini (acetal-PEG-b-PLA-vinyl). 
was performed for $1 \mathrm{~h}$. The resulting polymers were precipitated into cold 2-propanol, stored in a freezer over night, centrifuged at 10,500 rpm for $15 \mathrm{~min}$, and lyophilized in benzene. The number-average molecular weight of the block copolymer was determined by means of gel permeation chromatography (GPC) (column: TSKgel $\mathrm{G}^{3000 \mathrm{H}_{\mathrm{HR}}}$, TOSOH, Japan; eluent: $N, N$-dimethylformamide containing $10 \mathrm{mM} \mathrm{LiBr}$; flow: $1 \mathrm{ml} / \mathrm{min}$; column temperature: $40^{\circ} \mathrm{C}$ ) and ${ }^{1} \mathrm{H}-\mathrm{NMR}(\mathrm{ECX}-300,300 \mathrm{MHz}$, JEOL Ltd., Tokyo).

\subsection{Synthesis of PEG-PLA Block Copolymers Having Acetal- and Vinyl Termini (Acetal-PEG- $b$-PLA-Vinyl)}

PEG-PLA block copolymer having acetal- and vinyl termini (acetal-PEG- $b$-PLA-vinyl) was synthesized by

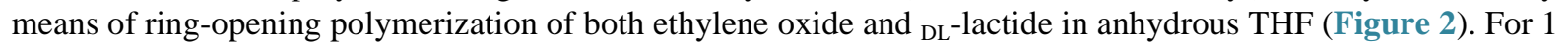
h, 3,3-diethoxypropanol, and potassium naphthalene were mixed in THF. The purified ethylene oxide was then added to the obtained potassium 3,3-diethoxypropioxide solution $(50 \mathrm{ml}$ ) and polymerization was carried out for $48 \mathrm{~h}$ at $25^{\circ} \mathrm{C}$. After polymerization, the purified $\mathrm{DL}^{-}$-lactide was added to the solution and the reaction was performed for $24 \mathrm{~h}$. After polymerization of ${ }_{\mathrm{DL}}$-lactide, methacrylic anhydride was added to the solution, and the reaction was performed for $4 \mathrm{~d}$. The resulting polymer was precipitated into cold 2-propanol, stored in a freezer over night, centrifuged at 10,500 rpm for $15 \mathrm{~min}$, and lyophilized in benzene. The number-average molecular weight of the block copolymer was determined by GPC (column: TSKgel G3000H $\mathrm{HR}_{\mathrm{HR}}$, TOSOH, Japan; eluent: $N, N$-dimethylformamide containing $10 \mathrm{mM} \mathrm{LiBr}$; flow: $1 \mathrm{ml} / \mathrm{min}$; column temperature: $40^{\circ} \mathrm{C}$ ) and ${ }^{1} \mathrm{HNMR}$ (ECX-300, 300 MHz, JEOL Ltd., Tokyo).

\subsection{Preparing Aldehyde-Terminated Polymeric Micelles That Have Noncrosslinked or Crosslinked Inner PLA Cores}

We dissolved the acetal-terminated PEG-PLA in $N, N$-dimethylacetamide and then performed dialysis against water by using a Spectra/Por7dialysis membrane (molecular weight cut-off: $1 \mathrm{kDa}$; Spectrum, Houston, TX, USA) for $24 \mathrm{~h}$. We added $\mathrm{HCl}$ to the polymeric micelle solution in order to adjust the $\mathrm{pH}$ to 2 . We mixed the solution for $2 \mathrm{~h}$ in order to convert the acetal group into an aldehyde group on the surface of the micelles. To stop the reaction, we adjusted the $\mathrm{pH}$ of the solution to 5 by adding an aqueous $\mathrm{NaOH}$ solution. The polymeric micelles that consisted of aldehyde-terminated PEG-PLA were finally obtained through dialysis against water for $24 \mathrm{~h}$, permitting the removal of the salt. To prepare the polymeric micelles having crosslinked inner PLA cores, we added acetal-PEG-b-PLA-vinyl instead of acetal-PEG-b-PLA-OH (in this case, ethylene dimethacrylate was added to the polymeric micelle solution for $1 \mathrm{~h}$, nitrogen was added for $30 \mathrm{~min}$, potassium persulfate was added, and then the crosslinking reaction was performed for $4 \mathrm{~d}$ ). The diameter distribution of the micelles was determined with dynamic light scattering (DLS), made possible by the Zetasizer Nano-ZS (Marvern Inst., UK).

\subsection{Evaluation of the Hydrogel-Formation Property}

We determined the hydrogel properties—-the storage modulus (G') and the loss modulus (G")—and the gelation time by using a rheometer (RS600, Thermo Electron, Germany, Sensor: PP35Ti). All measurements were performed at a gap of $0.4 \mathrm{~mm}$ and at a shear stress of $1.0 \mathrm{~Pa}$, with a frequency of $1 \mathrm{~Hz}$. A total of $0.2 \mathrm{ml}$ of the trilayered polymeric micelle solution $(30 \mathrm{w} / \mathrm{w} \%, \mathrm{pH} 5)$ and $0.2 \mathrm{ml}$ of the PEI solution (30 w/w\%, pH 6 - 11, molecular weight: $1800,10,000$, and 70,000) were mixed with a two-pronged needle, which is commonly used for mixing solutions of the commercially available tissue-adhesive BOLHEAL ${ }^{\mathrm{TM}}$ (Kaketsuken Co., Japan). Then the combined mixture was placed on the stage of the rheometer, and immediately thereafter, the measurement was started.

\section{Results and Discussion}

The obtained PEG-PLA block copolymers were characterized by ${ }^{1} \mathrm{H}$ NMR and GPC. The ${ }^{1} \mathrm{H}$ NMR spectra of the PEG-PLA block copolymers indicate that the block copolymers were synthesized successfully. Table 1 summarizes the results of the synthesis of the PEG-PLA block copolymers. The block copolymers with a code$\mathrm{OH}$ areacetal-PEG- $b$-PLA-OH, whereas the block copolymers with a code-vinyl areacetal-PEG- $b$-PLA-vinyl. The number-average molecular weights of the PEG and PLA units were determined on the basis of GPC and ${ }^{1} \mathrm{H}$ NMR, respectively. The number-average molecular weights, $M_{\mathrm{n}}$, of PEG and PLA were 1820 - 6000 and 1000 - 
Table 1. Characterization of synthesized poly (ethylene glycol)-block-poly (lactic acid) block copolymers (The block copolymers with a code-OH are acetal-PEG-b-PLA-OH, whereas the block copolymers with a code-vinyl is acetal-PEG-b-PLAvinyl. For example, the block copolymers with code 6-3-OH is an acetal-PEG-b-PLA-OH whose respective molecular wetighs of PEG and PLA are approximately 6000 and 3000).

\begin{tabular}{cccccc}
\hline \multirow{2}{*}{ Code } & \multicolumn{2}{c}{ Acetal-PEG } & \multicolumn{2}{c}{ PLA } & \multicolumn{2}{c}{ Acetal-PEG- $b$-PLA } \\
\cline { 2 - 5 } & $M_{\mathrm{n}}{ }^{1)}$ & $M_{\mathrm{w}} / M_{\mathrm{n}}{ }^{1)}$ & $M_{\mathrm{n}}{ }^{2)}$ & $M_{\mathrm{n}}{ }^{1), 2)}$ & $M_{\mathrm{w}} / M_{\mathrm{n}}{ }^{1)}$ \\
\hline $6-3-\mathrm{OH}$ & 6000 & 1.05 & 2900 & 8900 & 1.12 \\
$4-2-\mathrm{OH}$ & 4200 & 1.05 & 1800 & 6000 & 1.11 \\
$2-1-\mathrm{OH}$ & 2100 & 1.04 & 1000 & 3100 & 1.09 \\
$2-3-\mathrm{OH}$ & 1820 & 1.08 & 2900 & 4720 & 1.36 \\
$2-4-\mathrm{vinyl}$ & 2200 & 1.05 & 3750 & 5950 & 1.35 \\
\hline
\end{tabular}

${ }^{1)} \mathrm{GPC} ;{ }^{2) 1} \mathrm{H}$ NMR.

3750, respectively, and the distribution of molecular weight, $M_{\mathrm{w}} / M_{\mathrm{n}}$, was narrow.

We prepared polymeric micelles by using the obtained block copolymers. In preparing the polymeric micelle that would have crosslinked inner PLA core, we used acetal-PEG-b-PLA-vinyl instead of acetal-PEG-b-PLA$\mathrm{OH}$, where ethylene dimethacrylate and potassium persulfate made possible the crosslinking reaction. In the former case, after lyophilization of the resulting polymeric micelles, the micelles were not dissolved in $N, N$-dimethylacetamide, a suitable solvent for dissolving PEG-PLA block copolymers. The results clearly show that the crosslinking reaction was successfully performed. Figure 3 presents the diameter distribution of polymeric micelles prepared from acetal-PEG- $b$-PLA-OH or acetal-PEG-b-PLA-vinyl. Basically, in the case of acetalPEG-PLA-OH, the diameter of the resulting polymeric micelles increased as the molecular weight of the block copolymers increased. Unexpectedly and interestingly, the polymeric micelles prepared from acetal-PEG-PLA$\mathrm{OH}$ with codes 2-1-OH and 2-3-OH exhibited almost the same average diameter and diameter distribution, although the molecular weight of the former case's hydrophobic unit (PLA) was quite different from the molecular weight of the latter case's PLA (1000 and 2900, respectively). The results show that the two types of micelles to for it from each other regarding the packing of inner PLA cores; a polymeric micelle prepared from acetal-PEG-PLA-OH with codes 2-3-OH possessed a highly packed (i.e., well-entangled) inner PLA core, whereas a polymeric micelle prepared from acetal-PEG-PLA-OH with codes 2-1-OH possessed a lowly packed inner PLA core. Prepared from acetal-PEG-PLA-vinyl (code 2-4-vinyl), the inner-crosslinked polymeric micelle exhibited a slightly higher diameter than the block copolymer's molecular weight had led us to expect, due to the crosslinking formation in the inner PLA core after the addition of ethylene dimethacrylate.

Figure 4 presents a gelation of the hydrogel from a polyamine and a polymeric micelle. The hydrogel was rapidly formed when the polymeric micelle solution and the PEI solution were mixed under the optimal experimental conditions. We obtained a transparent hydrogel by mixing PEI and polymeric micelles together. The properties of hydrogels are affected by several factors, such as the $\mathrm{pH}$ of solutions and both the concentrations and the molecular weights of gel-forming components. At first, we estimated the effects that the polyamine, a main chain of the hydrogel, had on the hydrogel's gelation properties.

Table 2 summarizes the effects of both the PEI solution's $\mathrm{PH}$ and the PEI's molecular weight on the hydrogel's gelation properties. The rheometer gives the storage modulus ( $\left.G^{\prime}\right)$ and loss modulus ( $G$ ") that characterize the elastic and viscous characteristics of a material, respectively. $G^{\prime}$ is nearly equal to the complex modulus ( $G^{*}$ ), which reveals the degree of a material's elasticity, and, G' can be used as an indicator of gel strength. The gelation time is defined as the time when the solution phase changes from liquid-like behavior $\left(G^{\prime}<G^{\prime \prime}\right)$ to solid-like behavior ( $G^{\prime}>G^{\prime \prime}$ ) [32]. In order to avoid possible degradation of the PLA block in an alkaline solution, we used only the polymeric micelle solution at $\mathrm{pH}$. The storage modulus and the gelation time greatly depended on the $\mathrm{pH}$ of the PEI solutions and the molecular weight of PEI. The storage modulus increased as the $\mathrm{pH}$ of the PEI solution increased, whereas the storage modulus decreased as the $\mathrm{pH}$ of the PEI solution increased from 10 to 11 in some cases. The gelation time was reduced within $1 \mathrm{~s}$ as the $\mathrm{pH}$ of either the polymeric micelle or the PEI solutions increased. The time required for hydrogel formation (1 s) was comparable to or shorter than that previously reported for other tissue-adhesive hydrogels. The hydrogel was formed according to the Schiff base in a pH-dependent manner. Although the Schiff base-formation reaction generally proceeds faster at a slightly acidic 


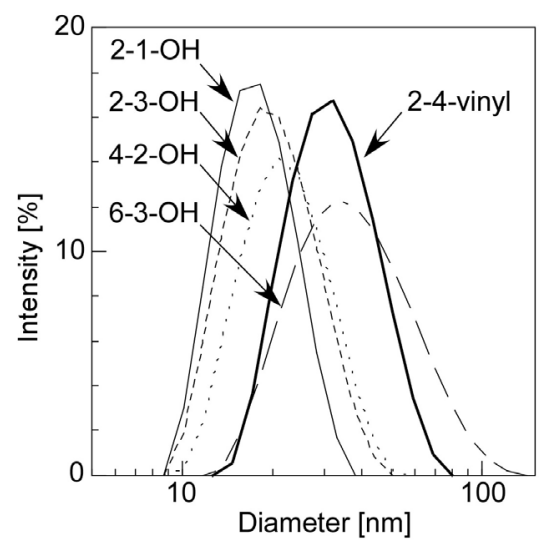

Figure 3. The diameter distribution of polymeric micelles prepared from acetal-PEG-b-PLA-OH or acetal-PEG-b-PLA-OH.

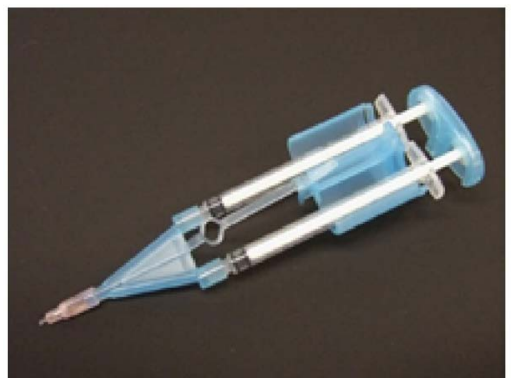

(a)

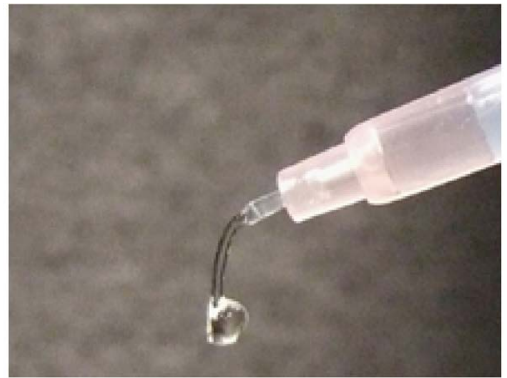

(b)

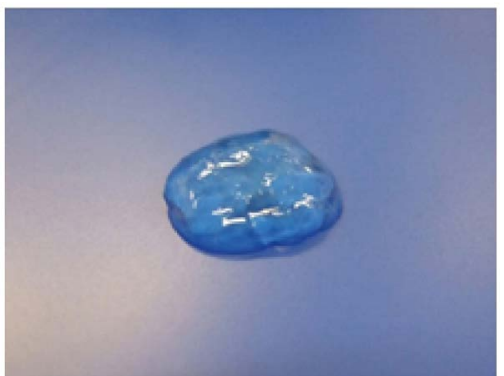

(c)

Figure 4. A gelation of the hydrogel from a polyamine and a polymeric micelle ((a) a two-pronged needle, which is commonly used for mixing solutions of the commercially available tissue-adhesive material; (b) hydrogel immediately after we mixed solutions containing either a polyamine or a polymeric micelle; and (c) a resulting hydrogel).

Table 2. The effects that the PEI solutions' pH and the molecular weight of PEI had on the hydrogel's gelation properties. Regarding the pairs of rows corresponding to the "pH of PEI solution” columns, the values in each pair's upper row show the storage modulus of the sample at $1 \mathrm{~h}(\mathrm{~Pa})$, whereas those in each pair's lower row (i.e., the values in parentheses) show the gelation time (s). Those entries that display only a dash (i.e., -) reflect the fact that no hydrogel was formed.

\begin{tabular}{|c|c|c|c|c|c|c|c|}
\hline \multirow{2}{*}{$\begin{array}{l}\text { Block copolymers used for } \\
\text { forming crosslinkable } \\
\text { polymeric micelles }\end{array}$} & \multirow{2}{*}{$M_{\mathrm{n}}$ of PEI } & \multicolumn{6}{|c|}{$\mathrm{pH}$ of PEI solution } \\
\hline & & 6 & 7 & 8 & 9 & 10 & 11 \\
\hline \multirow{3}{*}{$6-3-\mathrm{OH}$} & 70000 & $703(1)$ & $711(1)$ & $845(1)$ & $955(1)$ & $971(1)$ & $918(0)$ \\
\hline & 10000 & $249(1)$ & $307(1)$ & $388(1)$ & $634(1)$ & $615(1)$ & $242(1)$ \\
\hline & 1800 & 143 (98) & $96(324)$ & 207 (33) & $216(1)$ & $190(9)$ & $293(1)$ \\
\hline \multirow{3}{*}{$4-2-\mathrm{OH}$} & 70000 & $1840(1)$ & $1240(0)$ & $2050(0)$ & $2410(0)$ & $2690(0)$ & $1200(0)$ \\
\hline & 10000 & $1490(1)$ & $1660(1)$ & $749(1)$ & $1110(1)$ & $905(1)$ & $743(8.8)$ \\
\hline & 1800 & $14(422)$ & 257 (156) & 345 (26) & $415(1)$ & $305(1)$ & $400(1)$ \\
\hline \multirow{3}{*}{$2-1-\mathrm{OH}$} & 70000 & $710(1)$ & $1090(1)$ & $1400(1)$ & $1650(1)$ & $1460(1)$ & $2450(1)$ \\
\hline & 10000 & $580(1)$ & $480(1)$ & $576(1)$ & $740(1)$ & $1280(1)$ & $1400(1)$ \\
\hline & 1800 & $3(-)$ & $3(-)$ & $1(-)$ & $29(51)$ & $53(18)$ & $5(75)$ \\
\hline \multirow{3}{*}{$2-3-\mathrm{OH}$} & 70000 & 3860 (1) & $5530(1)$ & $6440(1)$ & $11300(1)$ & $12300(1)$ & $9550(1)$ \\
\hline & 10000 & 3110 (1) & $2170(1)$ & $3400(1)$ & $4700(1)$ & 4240 (1) & $6550(1)$ \\
\hline & 1800 & $226(1)$ & $584(1)$ & $1130(1)$ & $1420(1)$ & $727(1)$ & $362(1)$ \\
\hline
\end{tabular}


$\mathrm{pH}$ [33], the concentration of reactive $\mathrm{R}_{1}-\mathrm{NH}_{2}\left(\mathrm{R}_{1}-\mathrm{NH}_{3}{ }^{+}\right)$increases as $\mathrm{pH}$ increases. Thus, in the present study, the hydrogel rapidly formed as the $\mathrm{pH}$ of the PEI solutions increased. As the $\mathrm{pH}$ increased further, the enhanced Schiff base formation reaction made the PEI molecules cover the reactive surface of the polymeric micelles, decreasing the amount of the exposed aldehyde group on the micelles. Therefore, a white aggregate with low storage modulus was formed as the $\mathrm{pH}$ of the PEI solution increased. The storage modulus greatly depended on the $\mathrm{pH}$ of the solution used. Furthermore, when comparing the results for the acetal-PEG-PLA-OH involving codes 2-1-OH with the results for the acetal-PEG-PLA-OH involving codes 2-3-OH, we found that the latter exhibited a higher storage modulus than the former (gelation time-course is shown in Figure 5), although two types of crosslinkable polymeric micelles were almost identical to each other regarding diameter distribution as shown in Figure 3. In light of these results, the hydrogel that was formed from the polymeric micelles possessing a highly packed (i.e., well-entangled or crosslinked) inner core exhibited a higher storage modulus than the hydrogel that was formed from the polymeric micelles possessing a lowly packed structure. Furthermore, the crosslinking of the inner PLA core effectively contributed to the construction of a hydrogel exhibiting high gelation strength (Table 3). Comparing the results shown in Table 2 with those in Table 3, we found that the effects of inner PLA core's crosslinking on gelation strength was remarkable when the PEI solution's $\mathrm{pH}$ was low. Our results demonstrate that a microscopic structural difference among crosslinkers can induce a macroscopic change in the properties of the resulting hydrogels.

\section{Conclusion}

We prepared hydrogel that was formed from PEI acting as a main chain of the hydrogel and from polymeric micelle acting as both the crosslinker of the hydrogel and the container of the hydrophilic compounds. According to the results of our current study, one can change the viscoelastic property of this hydrogel by tuning the molecular weight and $\mathrm{pH}$ of the hydrogel's constituent components. Furthermore, the study's results suggest that the structural difference among crosslinkers affected the gelation property of the hydrogel. Interestingly and unexpectedly, hydrogel that was formed from the polymeric micelles possessing a highly packed (i.e., well-entangled or crosslinked) inner core exhibited a higher storage modulus than the hydrogel that was formed from the

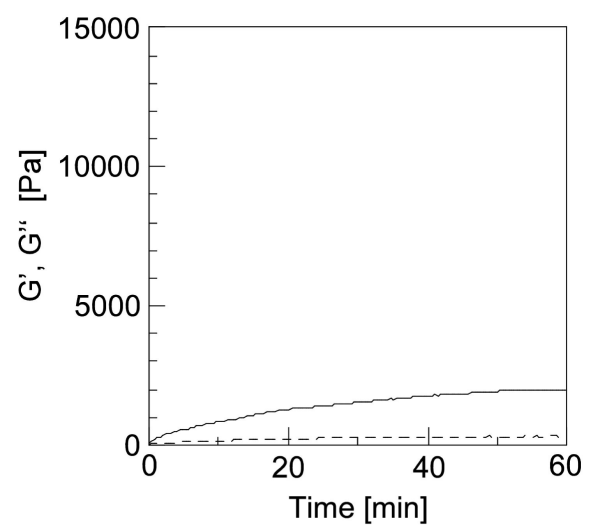

(a)

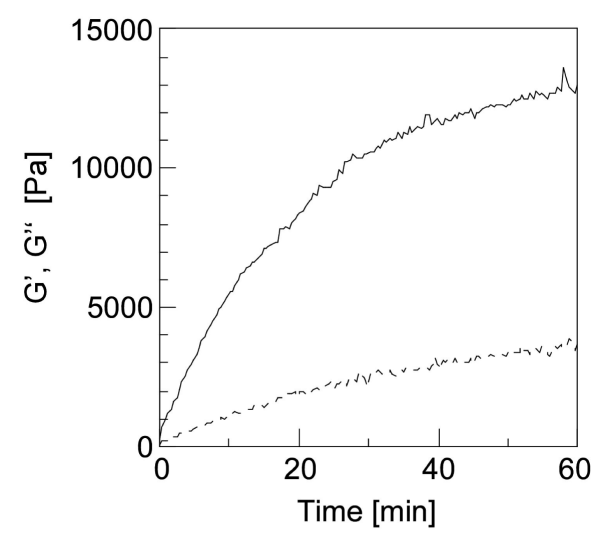

(b)

Figure 5. The gelation time-course when (a) acetal-PEG-PLA-OH (code 2-1-OH) and (b) acetal-PEG-PLA-OH (code 2-3$\mathrm{OH})$ were used as micelle-forming block copolymers. Solid and dotted lines represent storage modulus ( $\left.\mathrm{G}^{\prime}\right)$ and loss modulus (G"), respectively.

Table 3. The effects that the PEI solutions' pH and the molecular structure of bsock copolymer had on the hydrogel's gelation properties. Regarding the pairs of rows corresponding to the " $\mathrm{pH}$ of PEI solution" columns, the values in each pair's upper row show the storage modulus of the sample at $1 \mathrm{~h}(\mathrm{~Pa})$, whereas those in each pair's lower row (i.e., the values in parentheses) show the gelation time (s).

\begin{tabular}{cccccccc}
\hline \multirow{2}{*}{$\begin{array}{c}\text { Block copolymers used for forming } \\
\text { crosslinkable polymeric micelles }\end{array}$} & 6 & 7 & 8 & 9 & 10 & 11 \\
\cline { 2 - 6 } & $12700(0)$ & $6450(0)$ & $6930(0)$ & $11,600(0)$ & $11,200(9)$ & $7870(0)$ \\
\hline
\end{tabular}


polymeric micelles possessing a lowly packed structure. This phenomenon is attributable to the structural flexibility of polymeric micelles. In conclusion, our study suggests that a suitable selection of microscopic constituent components triggered changes in the properties of the resulting polymeric materials and that, in some cases, one can add unexpected macroscopic properties to the materials.

\section{References}

[1] Morikawa, T. (2001) Tissue Sealing. The American Journal of Surgery, 182, 29S-35S. http://dx.doi.org/10.1016/S0002-9610(01)00774-7

[2] MacGillivray, T.E. (2003) Fibrin Sealants and Glues. Journal of Cardiac Surgery, 18, 480-485. http://dx.doi.org/10.1046/j.0886-0440.2003.02073.x

[3] Czerny, M., Verrel, F., Weber, H., Muller, N., Kircheis, L., Lang, W., Steckmeier, B. and Trubel, W. (2000) Collagen Patch Coated with Fibrin Glue Components. Treatment of Suture Hole Bleedings in Vascular Reconstruction. The Journal of Cardiovascular Surgery, 41, 553-557.

[4] Turner, A.S., Parker, D., Egbert, B., Maroney, M., Armstrong, R. and Powers, N. (2002) Evaluation of a Novel Hemostatic Device in an Ovine Parenchymal Organ Bleeding Model of Normal and Impaired Hemostasis. Journal of Biomedical Materials Research (Applied Biomaterials), 63, 37-47. http://dx.doi.org/10.1002/jbm.10081

[5] Taguchi, T., Saito, H., Uchida, Y., Sakane, M., Kobayashi, H., Kataoka, K. and Tanaka, J. (2004) Bonding of Soft Tissues Using a Novel Tissue Adhesive Consisting of a Citric Acid Derivative and Collagen. Materials Science and Engineering: C, 24, 775-780. http://dx.doi.org/10.1016/j.msec.2004.08.037

[6] Fukunaga, S., Karck, M., Harringer, W., Cremer, J., Rhein, C. and Haverich, A. (1999) The Use of Gelatin-ResorcinFormalin Glue in Acute Aortic Dissection Type A. European Journal of Cardio-Thoracic Surgery, 15, 564-570. http://dx.doi.org/10.1016/S1010-7940(99)00084-6

[7] Kumar, A., Maartens, N.F. and Kaye, A.H. (2003) Evaluation of the Use of BioGlue in Neurosurgical Procedures. Journal of Clinical Neuroscience, 10, 661-664. http://dx.doi.org/10.1016/S0967-5868(03)00163-2

[8] Singer, A.J. and Thode, H.C. (2004) A Review of the Literature on Octyl Cyanoacrylate Tissue Adhesive. The American Journal of Surgery, 187, 238-248. http://dx.doi.org/10.1016/j.amjsurg.2003.11.017

[9] Ramakumar, S., Roberts, W.W., Fugita, O.E., Colegrove, P., Nicol, T.M., Jarrett, T.W., Kavoussi, L.R. and Slepian, M.J. (2002) Local Hemostasis during Laparoscopic Partial Nephrectomy Using Biodegradable Hydrogels: Initial Porcine Results. Journal of Endourology, 16, 489-494. http://dx.doi.org/10.1089/089277902760367458

[10] Nakayama, Y. and Matsuda, T. (1999) Photocurable Surgical Tissue Adhesive Glues Composed of Photoreactive Gelatin and Poly(Ethylene Glycol) Diacrylate. Journal of Biomedical Materials Research (Applied Biomaterials), 48, 511-521. http://dx.doi.org/10.1002/(SICI)1097-4636(1999)48:4\%3C511::AID-JBM17\%3E3.3.CO;2-M

[11] Ferland, R., Mulani, D. and Campbell, P.K. (2001) Evaluation of Sprayable Polyethylene Glycol Adhesion Barrier in a Porcine Efficacy Model. Human Reproduction, 16, 2718-2723. http://dx.doi.org/10.1093/humrep/16.12.2718

[12] Wallace, D.G., Cruise, G.M., Rhee, W.M., Schroeder, J.A., Prior, J.J., Ju, J., Maroney, M., Duronio, J., Ngo, M.H., Estridge, T. and Coker, G.C. (2001) A Tissue Sealant Based on Reactive Multifunctional Polyethylene Glycol. Journal of Biomedical Materials Research (Applied Biomaterials), 58, 545-555. http://dx.doi.org/10.1002/jbm.1053

[13] Buchta, C., Hedrich, H.C., Macher, M., Hocker, P. and Redl, H. (2005) Biochemical Characterization of Autologous Fibrin Sealants Produced by CryoSeal ${ }^{\circledR}$ and Vivostat ${ }^{\circledR}$ in Comparison to the Homologous Fibrin Sealant Product Tissucol/Tisseel $^{\circledR}$. Biomaterials, 26, 6233-6241. http://dx.doi.org/10.1016/j.biomaterials.2005.04.014

[14] Canonico, S. (2003) The Use of Human Fibrin Glue in the Surgical Operations. Acta Biomedica, 74, 21-25.

[15] Siedentop, K.H., Park, J.J., Shah, A.N., Bhattacharyya, T.K. and O’Grady, K.M. (2001) Safety and Efficacy of Currently Available Fibrin Tissue Adhesives. American Journal of Otolaryngology, 22, 230-235. http://dx.doi.org/10.1053/ajot.2001.24817

[16] Silver, F.H., Wang, M.C. and Pins, G.D. (1995) Preparation and Use of Fibrin Glue in Surgery. Biomaterials, 16, 891903. http://dx.doi.org/10.1016/0142-9612(95)93113-R

[17] Ciapetti, G., Stea, S., Cenni, E., Sudanese, A., Marraro, D., Toni, A. and Pizzoferrato, A. (1994) Cytotoxicity Testing of Cyanoacrylates Using Direct Contact Assay on Cell Cultures. Biomaterials, 15, 63-67. http://dx.doi.org/10.1016/0142-9612(94)90199-6

[18] Kaplan, M. and Baysal, K. (2005) In Vitro Toxicity Test of Ethyl 2-Cyanoacrylate, a Tissue Adhesive Used in Cardiovascular Surgery, by Fibroblast Cell Culture Method. The Heart Surgery Forum, 8, E169-E172. http://dx.doi.org/10.1532/HSF98.20041126

[19] Murakami, Y., Yokoyama, M., Okano, T., Nishida, H., Tomizawa, Y., Endo, M. and Kurosawa, H. (2007) A Novel Synthetic Tissue-Adhesive Hydrogel Using a Crosslinkable Polymeric Micelle. Journal of Biomedical Materials Re- 
search Part A, 80, 421-427. http://dx.doi.org/10.1002/jbm.a.30911

[20] Murakami, Y., Yokoyama, M., Nishida, H., Tomizawa, Y. and Kurosawa, H. (2008) A Simple Hemostasis Model for the Quantitative Evaluation of Hydrogel-Based Local Hemostatic Biomaterials on Tissue Surface. Colloids and Surfaces B: Biointerfaces, 65, 186-189. http://dx.doi.org/10.1016/j.colsurfb.2008.04.005

[21] Murakami, Y., Yokoyama, M., Nishida, H., Tomizawa, Y. and Kurosawa, H. (2009) In Vivo and in Vitro Evaluation of Gelation and Hemostatic Properties of a Novel Tissue-Adhesive Hydrogel Containing a Cross-Linkable Polymeric Micelle. Journal of Biomedical Materials Research (Applied Biomaterials), 91, 102-108. http://dx.doi.org/10.1002/jbm.b.31378

[22] Uchida, Y., Fukuda, K. and Murakami, Y. (2013) The Hydrogel Containing a Novel Vesicle-Like Soft Crosslinker, a “Trilayered” Polymeric Micelle, Shows Characteristic Rheological Properties. Journal of Polymer Science Part B: Polymer Physics, 51, 124-131. http://dx.doi.org/10.1002/polb.23187

[23] Moroishi, H., Yoshida. C. and Murakami, Y. (2013) A Free-Standing, Sheet-Shaped, "Hydrophobic" Biomaterial Containing Polymeric Micelles Formed from Poly(ethylene glycol)-Poly(lactic acid) Block Copolymer for Possible Incorporation/Release of "Hydrophilic” Compounds. Colloids and Surfaces B: Biointerfaces, 102, 597-603. http://dx.doi.org/10.1016/j.colsurfb.2012.08.050

[24] Ito, T., Yoshida. C. and Murakami, Y. (2013) Design of Novel Sheet-Shaped Chitosan Hydrogel for Wound Healing: A Hybrid Biomaterial Consisting of Both PEG-Grafted Chitosan and Crosslinkable Polymeric Micelles Acting as Drug Containers. Materials Science and Engineering: C, 33, 3697-3703. http://dx.doi.org/10.1016/j.msec.2013.04.056

[25] Zhang, Q., Wang, C.R., Babukutty, Y., Ohyama, T., Kogoma, M. and Kodama, M. (2002) Biocompatibility Evaluation of ePTFE Membrane Modified with PEG in Atmospheric Pressure Glow Discharge. Journal of Biomedical Materials Research, 60, 502-509. http://dx.doi.org/10.1002/jbm.1294

[26] Lee, H.J., Lee, J.S., Chansakul, T., Yu, C., Elisseeff, J.H. and Yu, S.M. (2006) Collagen Mimetic Peptide-Conjugated Photopolymerizable PEG Hydrogel. Biomaterials, 27, 5268-5276.

[27] Murakami, Y. and Hirata, A. (1999) Complex between $\alpha$-Chymotrypsin and Poly(ethylene glycol) Catalytically Active in Organic Media. Biotechnology Techniques, 13, 545-548. http://dx.doi.org/10.1023/A:1008987431361

[28] Murakami, Y. and Hirata, A. (1999) Poly(ethylene glycol)- $\alpha$-Chymotrypsin Complex Catalytically Active in Anhydrous Isooctane. Journal of Bioscience and Bioengineering, 88, 441-443. http://dx.doi.org/10.1016/S1389-1723(99)80224-2

[29] Murakami, Y., Hoshi, R. and Hirata, A. (2001) Borate Buffer Dramatically Enhances the Activity of Poly(ethylene glycol)- $\alpha$-Chymotrypsin Complex Catalytically Active in Anhydrous Isooctane Than Conventional Phosphate Buffer Even at Low Concentration. Biotechnology Letters, 23, 125-129. http://dx.doi.org/10.1023/A:1010345318275

[30] Murakami, Y., Hoshi, R. and Hirata, A. (2003) Characterization of Polymer-Enzyme Complex as a Novel Biocatalyst for Nonaqueous Enzymology. Journal of Molecular Catalysis B: Enzymatic, 22, 79-88. http://dx.doi.org/10.1016/S1381-1177(03)00009-2

[31] Murakami, Y. and Hirata, A. (1998) Enzymatic Synthesis of Peptides Review. Seibutsu-Kogaku Kaishi, 76, $238-254$.

[32] Ferry, J.D., Fitzgerald, E.R., Grandine, L.D. and Williams, M.L. (1952) Temperature Dependence of Dynamic Properties of Elastomers: Relaxation Distributions. Rubber Chemistry and Technology, 25, 720-729. http://dx.doi.org/10.5254/1.3543437

[33] Dohno, C., Okamoto, A. and Saito, I. (2005) Stable, Specific, and Reversible Base Pairing via Schiff Base. Journal of the American Chemical Society, 127, 16681-16684. http://dx.doi.org/10.1021/ja054618q 
Scientific Research Publishing (SCIRP) is one of the largest Open Access journal publishers. It is currently publishing more than 200 open access, online, peer-reviewed journals covering a wide range of academic disciplines. SCIRP serves the worldwide academic communities and contributes to the progress and application of science with its publication.

Other selected journals from SCIRP are listed as below. Submit your manuscript to us via either submit@scirp.org or Online Submission Portal.
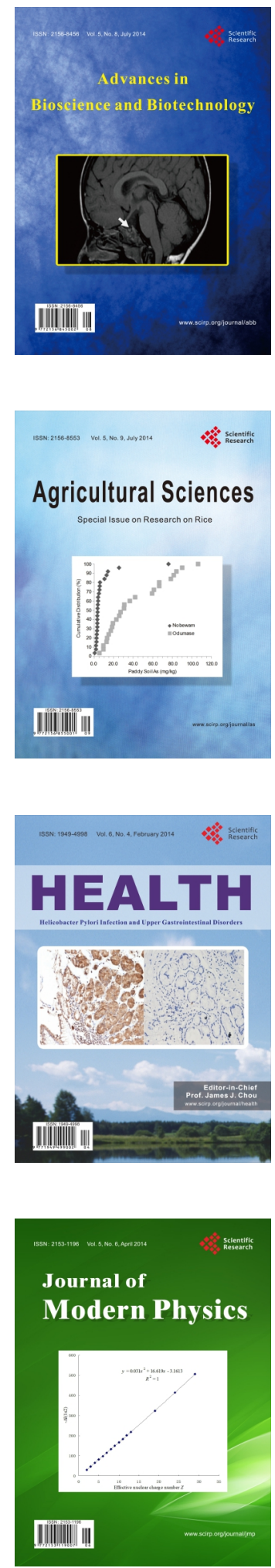
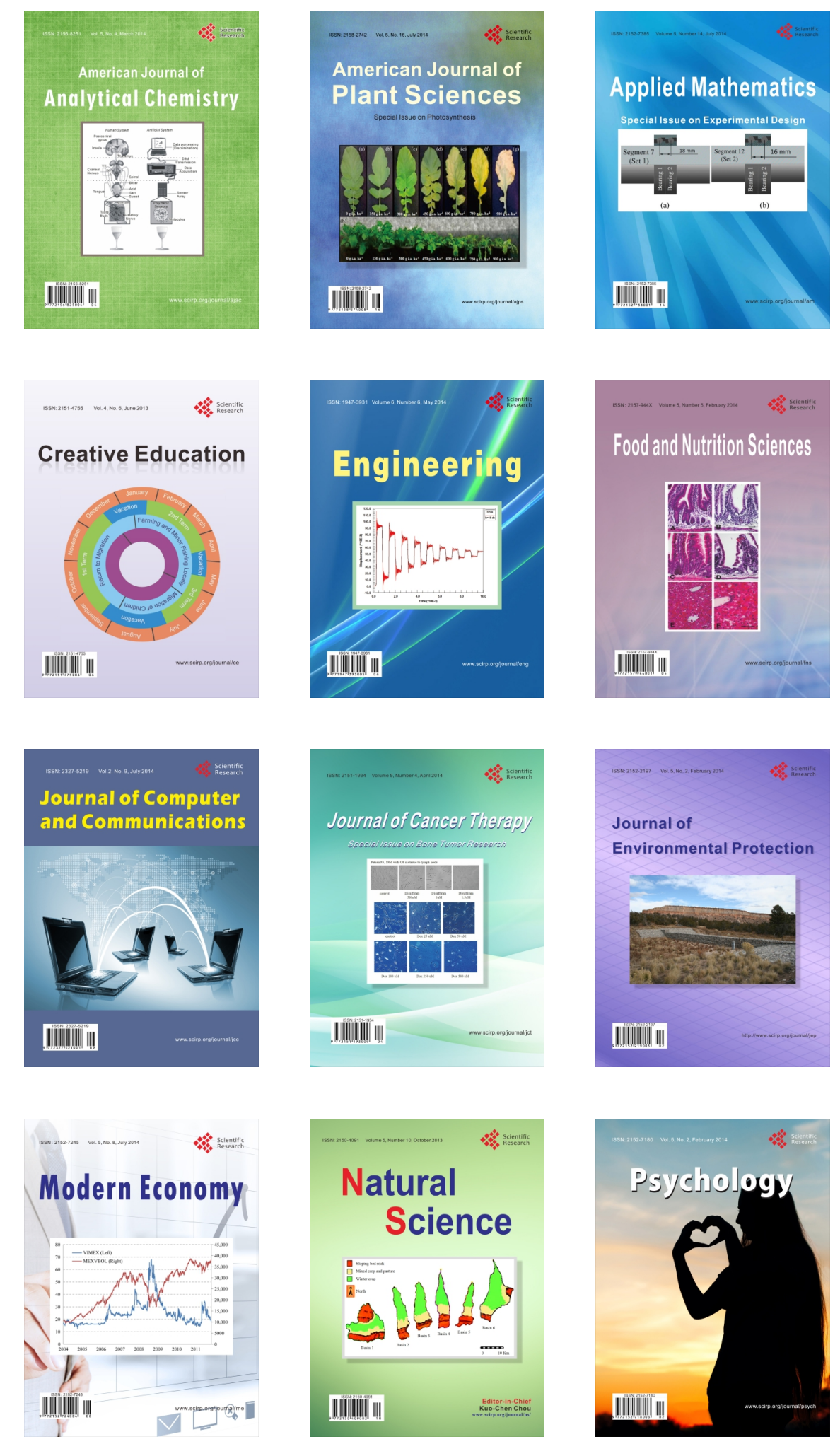